\title{
Test corpuscles in general relativity
}

\author{
By H. P. Robertson, Princeton University.
}

(Received 30th July, 1936. Read 6th November, 1936.)

\section{§1. Introduction.}

In the general theory of relativity, as in many other branches of theoretical physics, the material and energetical content of spacetime is considered, in the first instance, as an extended field, which is specified by means of field quantities (energy-momentum-stress tensor, charge-current density, electromagnetic field strength). From this point of view corpuscles (material particles, photons) are constructs obtained by first considering the field quantities as nonvanishing only within certain world tubes, and then passing by limiting processes to the idealisation in which these world tubes are shrunk into world lines. More precisely, this passage to the corpuscular description may be thought of as accomplished by replacing the original field by successive members of a sequence of field distributions, satisfying the same field laws, which cluster more and more in the neighbourhood of the world lines, and for which in some significant sense the total measure approaches a finite limit. Each such world line, together with the limiting measures of those portions of the field quantities associated therewith, is then a corpuscle; the form of the world line determines the motion of the corpuscle, and the associated "corpuscular quantities" its physical attributes (mass or energy, momentum, charge).

Only to the extent to which this programme is realisable are we justified in considering the physical content as representable by a corpuscular aggregate; thus if, as we now believe, both matter and radiant energy partake in undulatory properties, the programme can be successful only to that extent to which we may ignore the dispersive and diffractive phenomena unavoidably associated therewith. In the last analysis, then, a corpuscular description of the field is hedged about by restrictions originating in the inner structure of the field quantities; it would accordingly seem reasonable to believe that the more compatible any given aspect of their structure is with the above procedure, the more adequately it may be represented in such a description. 
Perhaps the most universal characteristic of the fields which crop up in modern theoretical physics is the existence in them of certain so-called conservation laws, expressed by the vanishing of the divergence of a field vector or tensor. These laws, as we shall see, do not of themselves contradict the programme outlined above, and we may therefore assume that they will be reflected in properties of the corpuscular description which are of significance whenever and to whatever extent such a description may be of value in the problem at hand We propose here to examine these implications of the conservation laws, particularly in the case in which the influence of that portion of the field in question on the kinematical structure of space-time may be ignored. The resulting construct will be called a test corpuscle, and the degree to which it may be taken to represent a physical entity will accordingly be circumscribed by this new limitation, in addition to those mentioned above as consequent to the inner structure of the field.

The problem with which we are here concerned may now be more precisely formulated as follows. Let $\mathrm{R}$ be a region of spacetime which is supplied, in some appropriate coordinate system $x^{i}(i=0,1,2,3)$, with a non-singular Riemannian metric

$$
d s^{2}=g_{i j} d x^{i} d x^{j}
$$

of signature -2 , whose coefficients $g_{i j}$ are considered as given functions which are of class one in their arguments $x^{i}$. There exists in $\mathrm{R}$ a vector $J^{k}$ or a tensor $T^{i k}$, whose components are likewise of class one, and which satisfies the "conservation" law

$$
\begin{aligned}
& J_{, k}^{k}=0, \quad \text { or } \\
& T^{i k}, k=0,
\end{aligned}
$$

where the index $k$, preceded by a comma, indicates convariant differentiation with respect to $x^{k}$. We propose here to discuss the kinematical and dynamical properties of the corpuscles resulting on replacing the field quantities $J^{k}, T^{i k}$, or some significant part thereof, by a singular distribution along one or more time-like or null world lines in R. Various aspects of this problem have been considered by previous writers, but we find it desirable to start afresh in order to obtain a precise formulation of the results, new and old, from a unified point of view; the connections with this previous work will be indicated during the course of the development. 


\section{§2. Kinematical preliminaries.}

We begin by specifying a preferred set of coordinate systems, which will facilitate our subsequent manipulation and interpretation. We note that an observer 0 , examining phenomena in the neighbourhood of an event $\mathrm{E}$ on his world line, will find it convenient to make a natural separation of this space-time neighbourhood into space and time. This he may do by referring the neighbourhood to coordinates $x^{i}$ in which the direction of the time axis at $\mathbf{E}$ coincides with the direction of his world line at the same event, and in which the directions of the three spatial axes are orthogonal to that of the time axis-and, if desired, to those of each other-in the sense of the metric (1.1). The remainder of the coordinate net may then be filled in quite arbitrarily, subject to the conditions laid down in $\S 1$ above. Thus $\mathrm{O}$ may, within wide limits, choose as the hypersurface $x^{0}=0$ a three-space $V$ having at each event a continuous time-like normal which coincides at $\mathrm{E}$ with the direction of his world-line, parameterise $\mathrm{V}$ by a set $x^{a}(a=1,2,3)$ of spatial coordinates, and augment the $x^{a}$ into a complete coordinate system $x^{i}$, within some closed region $\mathrm{R}$ containing $\mathbf{E}$ as an interior event, by employing as the temporal coordinate $x^{0} \equiv t$ the invariant interval $\int d s$ along geodesics which cut $\mathrm{V}$ orthogonally. The resulting "Gaussian" coordinate system is one in which the metric (1.1) assumes the simpler form

$$
d s^{2}=d t^{2}-d l^{2}, \quad d l^{2}=h_{a b}\left(x^{i}\right) d x^{a} d x^{b},
$$

where $d i^{2}$ is a positive definite metric whose coefficients $h_{a b}$ possess continuous derivatives with respect to their four arguments $x^{i}$. Furthermore, this choice of a Gaussian coordinate system can be made in such a way that it accomplishes a natural separation into space and time for a finite number of given observers at a finite number of points on their respective world lines, apart from limitations of a topological nature. In any case, the coordinate system thus set up does provide a separation at each event $E$ of $R$ which is natural, in the above sense, for some possible observer $O$ at $E$, and the transition from O's data concerning phenomena in the immediate neighbourhood of $\mathbf{E}$ to those of any other observer $0^{\prime}$ at $\mathrm{E}$ may then be accomplished as in the special theory of relativity.

It will suffice for our purposes to consider only space-time regions $\mathrm{R}$ which are four-cells, defined in terms of a given Gaussian coordinate system as follows: such an $R$ is the closed region bounded by two spaces $t=t_{1}, t=t_{2}\left(>t_{1}\right)$ and by the three-space generated by the 
geodesics $x^{a}=$ const. emanating from the boundary of a three-cell in the space $t=t_{1}$. A segment $\mathrm{L}$ of a world line will then be said to be contained in $\mathrm{R}$ provided it cuts the intersection of $\mathrm{R}$ with each space $t=$ const., $t_{1} \leqq t \leqq t_{2}$, in one interior point. Within $\mathrm{R}, \mathrm{L}$ will be defined by expressing the coordinates $x^{i}$ of events on it as continuous functions $\xi^{i}$, which we shall also assume are differentiable, of a parameter $p$ which increases monotonically with $t$; the tangent vector $u^{i}$ to $\mathrm{L}$ may then be defined in terms of the derivatives of $\xi^{i}$ :

$$
x^{i}=\xi^{i}(p), \quad u^{i}=\frac{d \xi^{i}}{d p} .
$$

The condition that $L$ be a time-like or null world line within $R$ then requires that

$$
u^{0} \geqq\left|\left\langle h_{a b} u^{a} u^{b}\right)^{\frac{1}{2}}\right|
$$

a corpuscle with a time-like world line (for which the inequality holds at each event) will be called a particle, and one with a null world line (the equality holding at each event) a photon. In the case of a particle we shall in general take as the parameter $p$ the arc-length $s$ measured in the direction of increasing $t$; the direction vector $u^{i}$ is then the unit vector, the components of which are the direction cosines of L. Finally, a corpuscle whose world line is such that a segment $\mathrm{L}$ of it is wholly contained within a closed cell $\mathrm{R}$ which contains no event of any other corpuscular world line, will be said to be isolated in $\mathrm{R}$.

We take as the total measure of a field quantity within a spatial region, preliminary to passing to the corpuscular description, its volume integral over the region. We shall, in particular, find it convenient to employ for this purpose regions in the spaces $t=$ const., whose volume element is given by

$$
d V \equiv h^{\frac{1}{4}}\left(t, x^{a}\right) d x^{1} d x^{2} d x^{3} .
$$

This expression is not, however, a scalar under general space-time transformations, and we shall therefore at times employ instead the quantity

$$
d V_{\pi} \equiv u^{0} d V
$$

in dealing with the differential neighbourhood of an event $\mathrm{E}\left(x^{i}\right)$ on a corpuscular world line $\mathrm{L}$, where $u^{0}$ is the temporal component of the vector $u^{i}$ tangent to $\mathrm{L}$ at $\mathrm{E}$. This alternative measure (2.5) is in fact a scalar under transformation from one Gaussian coordinate system to another, for it may be considered as the quotient of the space-time element $d t d V$ and the scalar $d p$. In case $\mathrm{L}$ is the world line of a 
particle, referred to the parameter $s, d V_{\pi}$ is the scalar measure of the projection of the spatial element $d V$ on to a space which cuts $\mathrm{L}$ orthogonally at $\mathrm{E}$-i.e. it is the scalar measure of the proper space of the particle in the neighbourhood of $E$. The unit of length is here taken to be such that the velocity of light is unity; thus no distinction is to be made between the unit of mass and the unit of energy.

\section{§3. Specification of corpuscular quantities.}

In the cases to be dealt with in the sequel, the conservation equations (1.2), (1.3) can be thrown into a form involving at most vectors (charge-current vector, ponderomotive force) and tensors (energy-momentum-stress tensor of material or radiation field) which are to be replaced, on transition to the corpuscular description, by the members of a sequence of quantities satisfying the same field laws, and which vanish in the limit at all events except those on the world lines of the corpuscles in question. In order to describe this situation in a form suitable for analytical manipulation, we adopt a Gaussian coordinate system $x^{i}$ and consider a segment $\mathrm{L}$ of a. corpuscular world line as contained within some four-cell $R$, in the sense defined in $\S 2$. We then consider $\mathrm{L}$ as contained within, and in a sense as the limit of, a nest of world tubes $T_{\kappa}(\kappa=1,2, \ldots)$ threading $R$ and having the properties

(a) each tube $T_{\kappa}$ contains within its interior its successor $T_{\kappa+1}$ and the segment $\mathrm{L}$.

(b) the intersection of $\mathrm{T}_{\kappa}$ with the space $t=$ const., $\left(t_{1}<t<t_{2}\right)$, is a closed three-cell within $R$, whose volume $V_{\kappa}$ and maximum diameter $D_{\kappa}$ both tend to the limit 0 as $\kappa \rightarrow \infty$.

For simplicity, we confine ourselves in this and the three succeeding sections to the consideration of a corpuscle which is isolated in $R$, in the sense defined in $\$ 2$; the amendments necessary for the later developments, in which the interaction between two or more portions of the field is best described in terms of the collision of two or more corpuscles, are for the most part self-explanatory. We may, then, allow each component $A\left(x^{i}\right)$ of the field quantities to be replaced in $\mathrm{R}$ by successive members of the set $A_{\kappa}\left(x^{i}\right),(\kappa=1,2, \ldots)$, in accordance with the condition

(c) for each value of $\kappa$, the quantity $A_{\kappa}\left(x^{i}\right)$ vanishes at all events $x^{i}$ outside the tube $T_{\kappa}$. 
Hence, by (a) and (b), for each event. $\mathrm{E}$ of $\mathrm{R}$, which is not on $\mathrm{L}$, there exists an integer $\kappa_{E}$ such that all "components of the field quantities constituting the set $\kappa \geqq \kappa_{E}$ vanish at $\mathrm{E}$.

The conditions (a)-(c) laid down above are merely in the nature of rules of procedure for the transition to the corpuscular description, and must now be augmented by more precise assumptions concerning the behaviour of the field quantities $A_{\kappa}\left(x^{i}\right)$ on allowing $\kappa \rightarrow \infty$; clearly the physical properties of the corpuscles are determined by this behaviour. Now each component $A\left(x^{i}\right)$ of the field densities represents some physical property of the field; we take as the total measure of this property for the field $A_{\kappa}$ contained within the tube $\mathrm{T}_{\kappa}$, at the $\kappa^{\mathrm{th}}$ stage in the transition, the volume integral of $A_{\kappa}$ over the intersection of $\mathrm{R}$ and the space $t=$ const. The limit $\alpha(t)$ of this integral as $\kappa \rightarrow \infty$ is then to be taken as the measure at time $t$ of the corresponding physical property of the corpuscle, as assigned by that virtual observer $\mathrm{O}$ at $\mathrm{E}\left(\xi^{0}=t, \xi^{a}\right)$ who has adopted the space-time separation implied by the Gaussian coordinate system $x^{i}$. We insure the existence of the limit $a$, and at the same time define implicitly the manner in which it is to be attained, by imposing the stronger requirement

(d) the sets $A_{\kappa}\left(x^{i}\right)$ of field quantities are to be so chosen that, on multiplication by an arbitrary continuous function $f\left(x^{i}\right)$ and integration over that portion of $t=$ const. within $\mathbf{R}$,

$$
\lim _{\kappa \rightarrow \infty} \int f\left(x^{i}\right) A_{\kappa}\left(x^{i}\right) d V=f\left(t, \xi^{a}\right) a(t),
$$

where $x^{i}=\xi^{i}$ is the event $\mathrm{E}$ in which the world line $\mathrm{L}$ cuts the space $t=$ const. Further, continuity or differentiability properties of $A\left(x^{i}\right)$ with respect to its arguments $x^{i}$ are to imply the same properties of $a(t)$ with respect to its argument $t$; we shall in general postulate at least continuity.

The definition of the corpuscular quantity $a(t)$ associated with a field quantity $A\left(x^{i}\right)$, which will always be denoted by the corresponding lower case Greek letter, is contained in (3.1) for $f \equiv 1$.

The corpuscular quantities $a^{i j}, \beta^{i}, \gamma$ which the observer 0 thus associates with a tensor field $A^{i j}$, a vector field $B^{i}$, and a scalar field $C$, respectively, are themselves not the components of a tensor, a vector, and a scalar at E. They are, however, closely related to 
such, for on multiplying the integral (3.1) by $u^{0}=d t ! d p$ and examining its behaviour in the neighbourhood of $E$, we find, with the aid of (2.5), that the resulting limiting quantities

$$
a^{i j} \equiv u^{0} \alpha^{i j}, \quad b^{i} \equiv u^{0} \beta^{i}, \quad c \equiv u^{0} \gamma
$$

are in fact the components of a tensor, a vector, and a scalar, respectively. These tensor quantities are not of the same direct physical significance to $\mathrm{O}$ as are the original corpuscular quantities; thus, in the case in which the construct is a particle whose world line is parameterised by its proper length $s$, they represent quantities obtained by integration over the proper space of the particle, whereas their indices refer to the space-time separation adopted by $O$. We shall, in fact, show that the tensor quantities of most significance in the corpuscular description, such as the energy-momentum vector or the charge, are obtained directly from the components of the pseudotensor quantities $a^{i j}, \beta^{i}$, and not from the associated tensors (3.2).

\section{\$4. Corpuscular implications of field conservation laws.}

We are now prepared to examine the implications, for the corpuscular description, of the conservation equations satisfied by the field quantities. These equations are characterised by the fact that each component of them may be thrown into the form

$$
\frac{1}{h^{\frac{1}{2}}} \frac{\partial}{\partial x^{k}}\left(h^{\frac{1}{3}} A^{k}\right)=B
$$

involving only field quantities $A^{k}, B$ (not necessarily a vector and a scalar) which are localisable in the manner contemplated in $\$ 3$ above; $h$ is here the determinant of the coefficients $h_{a b}$ of $d l^{2}$, equation (2.1). We therefore confine ourselves for the moment to a consideration of the consequences, from the corpuscular standpoint, of the form (4.1) common to these equations, reserving until later the more precise specification of $A^{k}$ and $B$.

The transition is now to be accomplished by replacing the field quantities $A^{k}, B$, in any space-time region $\mathrm{R}$ in which the corpuscle under discussion is isolated, by sets $A_{\kappa}^{k}, B_{\kappa}$ satisfying the same equation (4.1), and which define as $\kappa \rightarrow \infty$ a singular distribution along the world line segment $L$ in question, as prescribed by the conditions (a) -(d) of $\S 3$. We shall for simplicity assume that each component $A_{\kappa}^{k}$ is of class one-possessing continuous first derivatives-and that $B$ is, accordingly, continuous. In order to reap the full consequences of (4.1) under the conditions laid down in $\S 3$ for the transition, we 
first multiply this equation by an arbitrary function $f\left(x^{i}\right)$ of class one, and rewrite the resulting equation in the more significant form

$$
\frac{1}{h^{\frac{1}{2}}} \frac{\partial}{\partial x^{k}}\left(h^{\frac{1}{2}} f A^{k}\right)-f_{k} A^{k}-f B=0,
$$

where $f_{k}$ is the derivative of $f$ with respect to $x^{k}$. This new equation, as applied to the set $A_{\kappa}^{k}, B_{\kappa}$, is now to be multiplied by the volume element (2.4) and integrated over the portion of the space $t=$ const. within $R$. The contributions due to the terms $k=1,2,3$ in the first group in (4.2) may now, by Gauss' theorem, be transformed into surface integrals over the boundary of the intersection of $t=$ const. with $R$; we are then left with the integral form

$$
\frac{d}{d t} \int f A_{\kappa}^{0} d V-\int\left(f_{k} A_{\kappa}^{k}+f B_{\kappa}\right) d V=0,
$$

on interchanging the order of differentiation and integration in the first term.

The transition $\kappa \rightarrow \infty$, in accordance with the condition (d) above, now enables us to conclude from (4.3) that the corpuscular quantities $a^{k}(t), \beta(t)$, associated with $A^{k}\left(x^{i}\right), B\left(x^{i}\right)$ at time $t$, satisfy the equation

$$
\frac{d}{d t}\left(f a^{0}\right)-f_{k} a^{k}-f \beta=0
$$

where $f, f_{k}$ are to be evaluated at the event $\mathrm{E}\left(t, \xi^{a}\right)$ on $\mathrm{L}$. But the arguments $\xi^{i}$ of $f$, and therefore $f$ itself and its derivatives $f_{k}$, are defined implicitly as functions of $t$ by the condition $\xi^{0}=t$ and the equations (2.2) of L. On carrying out the differentiation indicated in the first term of (4.4), the left-hand side becomes a linear form in $f, f_{k}$ which must vanish identically for an arbitrary function $f$ of class one. Hence each coefficient in the form must vanish, and (4.4) may accordingly be replaced by the equivalent conditions

$$
\alpha^{k}=a^{0} \frac{d \xi^{k}}{d t}, \quad \frac{d \alpha^{0}}{d t}=\beta .
$$

Thus any four field quantities $A^{k}$, which satisfy an equation of the form (4.1), must be represented in the corpuscular description by four quantities $a^{k}$ which are proportional to the direction cosines of the (so far, given) corpuscular world line, where the factor of proportionality changes along the world line in a manner which is determined by the corpuscular quantity $\beta$ associated with the fifth field quantity $B$ appearing in the equation. 
We have now to examine the further consequences deducible from the above in the case in which the $A^{k}$ are the components of a contravariant vector, and in that in which they are the four components $A^{i k}\left(i\right.$ fixed, $k$ variable) defining the $i^{\text {th }}$ row of a contravariant tensor of degree two. In the first of these cases (4.1) is simply the continuity equation

$$
A^{k},{ }_{k}=B,
$$

where $B$ is a scalar. The only remark which we have here to add to our previous discussion is that the temporal component

$$
a^{0} \text { is a scalar; }
$$

this result follows from the first set of equations (4.5) on multiplication by $u^{2}$, for by (3.2) the left-hand side $u^{0} \alpha^{k}$, and therefore the right-hand side $a^{0} u^{k}$, is a vector, and since $u^{k}$ is itself a vector the factor of proportionality $a^{0}$ must be a scalar.

Consider next the tensorial equation

$$
A^{i k},{ }_{k}=P^{i},
$$

where $A^{i k}$ is a contravariant tensor of degree two and $P^{i}$ a contravariant vector. The $i^{\text {th }}$ component of (4.8) is an equation of precisely the form (4.1), where

$$
A^{k}=A^{i k}, \quad B=P^{i}-\left\{\begin{array}{c}
i \\
j k
\end{array}\right\} A^{j k}
$$

the Christoffel symbols of the second kind here involved are computed from the metric (2.1). Equations (4.5) then become, under the conditions (a)-(d) governing the transition,

$$
a^{i k}=a^{i 0} \frac{d \xi^{k}}{d t}, \quad \frac{d a^{i 0}}{d t}+\left\{\begin{array}{c}
i \\
j k
\end{array}\right\} a^{j k}=\pi^{i},
$$

where $a^{i k}, \pi^{i}$ are the corpuscular quantities associated with $A^{i k}, P^{i}$ and the Christoffel symbols are evaluated at the event $\mathrm{E}\left(t, \xi^{a}\right)$ on $\mathrm{L}$. The first set of equations (4.10) implies that the matrix $\left(a^{i k}\right)$ is at most of rank one, and that the four quantities

$$
a^{i 0} \equiv a^{i}
$$

are the components of a contravariant vector, as can be shown by a direct examination of their law of transformation-or seen more readily from the fact that

$$
a^{i k}=u^{0} a^{i k}=a^{i 0} u^{k}
$$

are, by (3.2), the components of a tensor of degree two. The 
fundamental equations (4.10) may, on multiplication by $u^{0}$, be thrown into the tensorial form

$$
a^{i k}=a^{i} u^{k}, \quad \frac{d a^{i}}{d p}+\left\{\begin{array}{c}
i \\
j k
\end{array}\right\} a^{j} u^{k}=p^{i},
$$

where $p^{i}$ is the vector associated with $\pi^{i}$ as in (3.2). Here, again, the corpuscular consequences of the field equations (4.8) imply no essential restriction on the form of the world line $L$ of the corpuscle; they merely state that, given the corpuscular world line $\mathrm{L}$, there exists a vector $a^{i}$, which together with the direction cosines $u^{k}$ of $\mathrm{L}$ defines $\alpha^{i k}$, and whose vectorial rate of change along $L$ is determined by the vector $p^{i}$ associated with the given $P^{i}$.

This latter situation is radically changed if we require in addition that $A^{i k}$ be a symmetrical tensor-i.e. that $A^{i k}=A^{k i}$. For it then follows immediately from (4.12) that the vector $a^{i}$ falls along the tangent $u^{i}$ to $\mathrm{L}$, and we may accordingly write

$$
a^{i}=a u^{i}
$$

where $a$ is a new scalar. The second set of equations (4.12) then assumes the form

$$
a\left[\frac{d u^{i}}{d p}+\left\{\begin{array}{c}
i \\
j k
\end{array}\right\} u^{j} u^{k}\right]=p^{i}-u^{i} \frac{d a}{d p},
$$

where as usual $u^{i}=d \xi^{i} / d p$. These equations will be interpreted in the sequel as placing an essential restriction on the form of the corpuscular world line $\mathrm{L}-i . e$. on the motion of the corpuscle.

\section{§5. Motion of a test particle.}

As a first application of the methods developed above, we consider the problem of the motion of an isolated test particle in general relativity. Most of the results we here derive have been obtained, in some form or other, by previous investigators ${ }^{1}$, but it may not be superfluous to treat them anew from the standpoint adopted in the present attack. Consider, then, the "conservation" equations

$$
T^{i k}{ }_{k}=0
$$

1 A bove all, by $Н$. Weyl in his important treatise Raum-Zeit-Materie; we shall here refer to the 5th edition of this work, although many of the results are to be found in the earlier editions. They are also obtained, although from a less fundamental standpoint, in the standard English work, A. S. Eddington, The Mathematical Theory of Relativity (Cambridge, 1923). 
in a region $R$ in which the total energy-momentum-stress tensor

$$
T^{i k} \equiv M^{i k}+S^{i k}
$$

may be separated, at least phenomenologically, into an inertial tensor $M^{i k}$ and a tensor $S^{i k}$ representing the external field. The first of these, which is to characterise the particle itself on transition to the corpuscular description, is to be localisable in the neighbourhood of a time-like world line $\mathrm{L}$ in $\mathrm{R}$ and is to satisfy the conditions

$$
M^{00} \geqq 0, \quad g_{i k} M^{i k} \geqq 0
$$

non-trivially, in the sense that the corpuscular quantities $\mu^{00}, g_{i k} \mu^{i k}$ associated therewith shall be positive. Of the second part, $S^{i k}$, we require merely that the power-force vector

$$
P^{i} \equiv-S^{i k}, k
$$

obtained therefrom be localisable in the neighbourhood of $\mathrm{L}$; the associated corpuscular vector $p^{i}$ will then be interpreted as the force acting on the particle due to the external field. We do not assume, for the moment, that the stress tensors are symmetrical in their indices $i$ and $k$.

The conservation equations (5.1) are now of the form (4.8), where $A^{i k}$ is the inertial tensor $M^{i k}$ and $P^{i}$ is the force vector (5.4). Since the world line $\mathrm{L}$ is assumed time-like at each event in $R$, we may choose as its parameter $p$ the proper time $s$ measured along it, and the corpuscular consequences (4.12) of the conservation laws assume the form

$$
m^{i k}=m^{i} u^{k}, \quad \frac{d m^{i}}{d s}+\left\{\begin{array}{c}
i \\
j k
\end{array}\right\} m^{j} u^{k}=p^{i},
$$

where $m^{i}$ is the vector $\mu^{i 0}$ and $u^{k}$ is the "direction cosine" $d \xi^{k} / d s$ of L. Now $m^{0}(>0)$ is interpreted as the mass of the particle at the event $\mathrm{E}$, as viewed by the virtual observer $\mathrm{O}, x^{a}=$ const., at $\mathrm{E}$, for it is the total measure $\mu^{00}$ obtained by integrating the mass density $M^{00}$ over the proper space of $O$ in the neighbourhood of $E$ and passing to the corpuscular idealisation; similarly, the spatial components $m^{a}$ are O's measure of the momentum of the particle when it is at $\mathrm{E}$. Thus we see how mass (or energy) and momentum (or rate of flow of energy), which are represented in the field by the components $M^{i 0}$ of a tensor, are represented in the corpuscular description by the massmomentum vector $m^{i}$. Assuming the power-force vector $p^{i}$ as given along the path $\mathrm{L}$ of the particle, the second set of equations (5.5) merely tell us how $m^{i}$ is propagated along the given world line $\mathrm{L}$; 
these equations are thus the invariantive four-dimensional formulation "proper rate of change of momentum along $L$ equals force" of the second law of motion, together with the corollary "proper rate of change of energy equals power."

In order to complete our corpuscular dynamics, we must specify how the dynamical concept " momentum" $m^{i}$ is related to the kinematical concept "velocity," here represented by $u^{i}$; this question, which has been raised explicit]y by Eddington ${ }^{1}$, is here to be answered in terms of the symmetry of the inertial tensor $M^{i j}$ with respect to its indices. For if, as is generally assumed from the beginning, $M^{i j}$, and therefore also its corpuscular counterpart $\mu^{i j}$, is symmetrical, it follows as in (4.13) that

$$
m^{i}=m u^{i}, \quad(m>0),
$$

where the scalar magnitude $m$ of $m^{i}$ is the proper or rest mass of the particle. Thus under these conditions the energy-momentum vector $m^{i}$ must coincide in direction with the velocity four-vector $u^{i}$-and, conversely, if this latter situation is to obtain, at least the limiting measure $\mu^{i j}$ of the inertial field must be symmetrical in its indices.

The second set of equations (5.5) now yield the equations of motion

$$
m\left[\frac{d^{2} \xi^{i}}{d s^{2}}+\left\{\begin{array}{c}
i \\
j k
\end{array}\right\} \frac{d \xi^{j}}{d s} \frac{d \xi^{k}}{d s}\right]=p^{i}-\frac{d \xi^{i}}{d s} \frac{d m}{d s} .
$$

Since $u^{i}=d \xi^{i} / d s$ is here a unit vector, it is orthogonal to its contravariant rate of change, given by the expression in brackets above, and hence on multiplying these equations by the covariant component $u_{i}$ and summing over $i$ we find

$$
\frac{d m}{d s}=u_{i} p^{i}
$$

the proper rate of change of proper mass (or energy) is equal to the proper rate at which work is done by the external field. We have with this shown that the field conservation laws imply, under the conditions (a)-(d) laid down in $\S 3$ and the assumption that the inertial tensor is symmetrical, that the corpuscular trajectory must satisfy the second order equations of motion (5.7). It remains only to apply this general result to cases in which the force vector $p^{i}$ represents situations of particular physical interest.

Op. cit., p. 125. 
We consider first the case in which $T^{i k}$ consists only of the inertial part $M^{i k}$; we are then dealing with an isolated particle subject to no explicit external field. The force vector $p^{i}$ vanishes, and by (5.8) the proper mass $m$ of the particle is constant along its trajectory. The equations (5.7) then yield the familiar result that the motion of a free neutral test particle is along a geodesic of the metric (1.1)1.

As a second illustration, we consider the motion of a charged particle in an electromagnetic field. In accordance with the phenomenological electrodynamics incorporated into the general theory of relativity, we may describe the total field as in (5.2)-(5.4), where the ponderomotive force

$$
P^{i}=-F_{k}^{i} J^{k}
$$

is derived from the exterior electromagnetic field $F_{i k}$ and the chargecurrent vector $J^{k}$ of that portion of the field which is to give rise to the charged particle. Now the vector $J^{k}$ is a localisable vector which satisfies, in this theory, the conservation equation (1.2), and hence its corpuscular counterpart $\iota^{k}$ obeys the equations (4.5). Further, by (4.7), the temporal component $\iota^{0}$ is a scalar, the charge $e$ of the particle as measured by any observer at the event $\mathrm{E}$ in question; thus charge, in contradistinction to "mass" $m^{0}$, is independent of the relative motion of particle and observer. In terms of the vector $j^{k}$ associated with $\iota^{k}$ as in (3.2), equations (4.5) may now be expressed in the form

$$
j^{k}=e u^{k}, \quad e=\text { const. }
$$

Equation (5.9) yields, on integration over the cross-section $t=$ const. and multiplication by $u^{0}$, the expression

$$
p^{i}=-e F_{k}^{i} u^{k}
$$

for the ponderomotive force on the particle. Since $F_{i k}$ is an antisymmetrical tensor, the sum $u_{i} p^{i}$ vanishes, and hence by $(5.8)$ the proper mass $m$ of the particle is again a constant of the motion. The equations (5.7), (5.11) are then the usual equations of motion of a charged particle under the influence of an external electromagnetic field $F_{i k}^{2}$.

Our derivation of these results differs from that of Weyl or Eddington mainly in its formulation of the postulate concerning the behaviour of the energy-momentum-stress tensor on the transition to

1 Weyl, op. cit., §38; Eddington, op. cit, $\$ 56$.

2 Weyl, op. cit., p. 285 ; Eddington, op. cit., p. 190. 
the corpuscular description. Thus these authors assume, in addition to indicial symmetry, the rotational symmetry of the elementary particles, and are thereby enabled to conclude the relation (5.6) between momentum and velocity; from our standpoint this is accomplished entirely by the general postulate (d), which would almost seem inherent in the very notion of the transition ${ }^{1}$. Our methods allow us to deal directly with the full problem, without introducing the concept of the "exterior field" due to the Riemannian character of the metric, as in Weyl's treatment, and can be applied, as shown in the succeeding section, to the case in which the corpuscles are light quanta. On the other hand, we have not attempted here to take into account the effect of the corpuscles in question on the metric field, as has been done by $\mathrm{Weyl}^{2}$ or, from another standpoint, by C. Lanczos ${ }^{3}$; it would seem, however, that such an investigation, which is beyond the scope of the present paper, could be accomplished along substantially the same lines.

\section{§6. Motion of a test photon.}

We come now to the case of most novel interest in the present investigation, that in which the test corpuscle is obtained from an energy-momentum-stress tensor

$$
T^{i k}=S^{i k}, \text { where } g_{i k} S^{i k}=0, S^{00} \geqq 0 .
$$

This situation arises naturally in dealing with a field which is purely electromagnetic in the region $R$ under examination, and we accordingly refer to the resulting corpuscular constructs as photons. It is to be emphasised, however, that at least in the preliminary stages no reference is made to the inner structure of $S^{i k}$, other than that its divergence and its trace both vanish.

The vanishing of the divergence of the localisable tensor $\mathcal{S}^{i k}$ implies, by (4.10), that the associated corpuscular quantities $\sigma^{i j}$ satisfy the equations

$$
\sigma^{i k}=s^{i} \frac{d \xi^{k}}{d t}, \quad \frac{d s^{i}}{d p}+\left\{\begin{array}{c}
i \\
j k
\end{array}\right\} s^{j} \frac{d \xi^{k}}{d p}=0,
$$

where $p$ is any suitable parameter and we have written $s^{i} \equiv \sigma^{i 0}$, in

1 The necessity of demanding that the limiting "mass-centre" of the field fall no the path $\mathrm{L}$, which is here insured by our formulation of (d), has been pointed out by A. Einstein and W. Mayer in a discussion with the writer.

2 Op. cit., p. 283.

3 Zeits. f. Phys. 59, p. 514 (1930). 
accordance with (4.11). Further, by (6.1) and the rules governing the transition, the trace of $\sigma^{i k}$ must vanish, whence

$$
s_{i} u^{i}=0, \text { where } u^{i}=\frac{d \xi^{i}}{d p} .
$$

Without imposing conditions on the symmetry of $S^{i k}$, we see that the conservation equations and the vanishing of the trace require that, whatever the path $\mathrm{L}$, the energy-momentum vector $s^{i}$ must be orthogonal to it and be transported along it by parallel displacement.

Although the investigation of the relation between $s^{i}$ and $u^{i}$ under specific unsymmetrical tensors $S^{i k}$ (such as that of Minkowski) might be of interest in other connections, we are here concerned primarily with a radiation field in otherwise free space, and therefore adopt the usual condition that $S^{i k}$ be symmetrical in its indices $i$ and $k$. But then, as in (4.13), $s^{i}$ is proportional to $u^{i}$; we here find it convenient to choose the parameter $p$ in such a way that these two vectors are identical-specifically, we define

$$
p \equiv \int d t / s^{0}, \text { whence } s^{i}=s^{0} \frac{d \xi^{i}}{d t}=\frac{d \xi^{i}}{d p}=u^{i} .
$$

The equations (6.2), (6.3) for the parallel transport of $s^{i}$ are then the equations

$$
\frac{d^{2} \xi^{i}}{d p^{2}}+\left\{\begin{array}{c}
i \\
j k
\end{array}\right\} \frac{d \xi^{j}}{d p} \frac{d \xi^{k}}{d p}=0, \quad u_{i} u^{i}=0
$$

of the null geodesics of the metric (1.1) ${ }^{1}$ We have with this proved the assumptions of Kermack, McCrea and Whittaker ${ }^{2}$ that the path of a light pulse is a null geodesic, along which the energy-momentum vector $s^{i}$ is carried by parallel transport. The results derived therefrom by these authors, in particular that as a consequence of (6.4), (6.5) a photon description of light is possible also in the space-times

1 Cf. the work of O. Halpern, Phys. Rev. 48, p. 431 (1935), where it is shown that, on the classical theory, a plane wave field, whose energy-momentum-stress tensor is symmetrical and satisties the condition (6.1), is necessarily propagated with the velocity of light.

${ }^{2}$ Proc. Roy. Soc. Edin. 53, p. 43 (1932). Cf. also J. L. Synge, Quart. Journ. Math. (Oxford) 6, p. 199 (1935), who shows that their assumption of the parallel transport of $s^{i}$ can be replaced by energy-momentum considerations concerning the interaction of the material source and receiver in establishing the possibility of a photon description in general relativity; the justification of these assumptions, from the present standpoint, is considered in the succeeding $\$ 7$. 
contemplated in the general theory of relativity, then follow as in their work.

For the sake of completeness, we add here a derivation, based on their theorem (2.6) concerning neighbouring null geodesics, of the fact that the energy $s^{0}$ of such a photon is proportional to a frequency $\nu$ intrinsically associated with the phenomenon; this result serves as a bridge between the corpuscular or geometrical optics, to which we have so far confined our considerations, and the more fundamental wave or physical optics. The theorem under discussion states that for any two neighbouring null geodesics the quantity

$$
J \equiv u_{i} \delta \xi^{i}
$$

is a constant of the motion, where $u^{i}$ is the transport vector of one of the null geodesics and $\delta \xi^{i}$ is the displacement between associated events on the two paths; this association is arbitrary within wide limits, for if we add to $\delta \xi^{i}$ any infinitesimal displacement along the path, the value of $J$ is unchanged in terms of first order. Consider now light impulses sent out with a frequency $\nu_{0}$ by an observer $O_{0}$ at $x^{a}=x_{0}^{a}$, const., and received by an observer $O_{1}$ stationed at $x^{a}=x_{1}^{a}$, const. Then if $\nu_{1}$ be the frequency with which these impulses are received by $O_{1}$, we may say that the $\nu_{0} \delta t_{0}$ impulses sent out by $\mathrm{O}_{0}$ in the interval $t_{0}, t_{0}+\delta t_{0}$ are received by $\mathrm{O}_{1}$ in the interval $t_{1}$, $t_{1}+\delta t_{1}$, where

$$
\nu_{0} \delta t_{0}=\nu_{1} \delta t_{1}
$$

On applying (6.6) to the two null geodesics defining this signal bandi.e. to the null geodesics which originate at $x_{0}^{a}$ at times $t_{0}, t_{0}+\delta t_{0}$, and pass through $x_{1}^{a}$ at times $t_{1}, t_{1}+\delta t_{1}$, respectively-we find

$$
\left(u^{0} \delta t\right)_{0}=\left(u^{0} \delta t\right)_{1} \text {, }
$$

where we have taken account of the fact that for the metric (2.1) there is no distinction between the covariant component $u_{0}$ and the contravariant component $u^{0}$ of the transport vector. On noting that $u^{0}=s^{0}$ and comparing our last result with (6.7), we find that

$$
s^{0} / \nu \text { is a constant of the motion. }
$$

Now since, as shown by the authors quoted above, this Doppler frequency $v$ may be identified with the optical frequency of the pulse-again within the limitations dwelt upon in the Introductionwe have established the compatibility of our considerations with Planck's law

$$
s^{0}=h \nu,
$$


where $h$ is the universal constant of action-and hence with the possibility of a photon description of the radiation field in general relativity.

We have with this justified, from first principles, the procedure adopted by Hubble and Tolman ${ }^{1}$ in deriving their expression for the luminosity of distant nebulae in cosmological space-times ${ }^{2}$. For not only is the luminosity of a receding source decreased by the factor $(1+\delta \lambda / \lambda)^{-1}$ due to weakening by Doppler effect, but also again by the same factor due to the slower rate of reception of photonscontrary to the contention of de Sitter $^{3}$ that this factor should be applied but once.

\section{§7. Collision phenomena. Compton effect.}

As a final application of the methods developed above, we treat briefly the case in which two or more portions of the field $T^{i k}$ interact in a manner which is best described, from the corpuscular standpoint, as a collision between two or more particles. It will suffice to consider two corpuscles A, B whose world lines intersect at an event $\mathbf{E}\left(\xi^{i}\right)$, and are otherwise isolated, from one another and from other corpuscles, within a four-cell $R$ containing $E$ in its interior. Let the segments $L_{A}, L_{B}$ of their world lines within $R$ be enclosed within two nests of world tubes, as prescribed by the conditions (a) and (b) of $\S 3$, in such a way that the intersections of these two nests with the boundary $t=t_{1}$, and with the boundary $t=t_{2}$, of $\mathrm{R}$ have in each case no common event. The two portions of the field $T^{i k}$, whose interaction within $R$ is to be described as the collision, are now to be replaced within $R$ by a series of field distributions which satisfy the remaining conditions (c) and (d) of $\S 3$ in the neighbourhoods of the bounding hypersurfaces $t=t_{1}, t=t_{2}$; in addition, each set $T_{k}^{i k}$ of the total field distribution is to satisfy the conservation equations (1.3) whose consequences we now examine.

The fundamental equations (1.3) satisfied by $T_{\kappa}^{i k}$ may be written in the form

$$
\frac{1}{h^{\frac{1}{2}}} \frac{\partial}{\partial x^{k}}\left(h^{\frac{1}{2}} T_{\kappa}^{i k}\right)=-\left\{\begin{array}{c}
i \\
j k
\end{array}\right\} T_{\kappa}^{j k},
$$

1 Astrophys. Journ. 82, p. 302 (1935).

2 This case, considered in detail by Kermack, McCrea and Whittaker, can also be treated with the aid of the results obtained by M. v. Laue. Sitzungsber. preuss. Akud. Wiss. 1931, p. 123 . Note added in proof: Such a treatment, by v. Iaue himself, has since appeared in Zeits. f. Astrophys. 12, p. 208 (1936).

${ }^{3}$ Bull. Astronom. Inst. Netherlands 7, No. 261, p. 210 (1934). 
where $h$ is the determinant of the coefficients $h_{a b}$ of the auxiliary metric $d l^{2},(2.1)$. On multiplying these equations by the element of volume $d t d V$ of space-time and integrating over the region $\mathbf{R}$ we find that

$$
\left[\int T_{\kappa}^{i 0} d V\right]_{1}^{2}=-\int_{t_{1}}^{t_{3}} d t \int\left\{\begin{array}{c}
i \\
j k\}
\end{array}\right\} T_{\kappa}^{j k} d V,
$$

where the term on the left is the difference of the spatial integral over the boundaries $t=t_{2}, t=t_{1}$ of $\mathrm{R}$, and we have again made use of the fact that the integrals arising from the remaining terms $k=1,2,3$ in the divergence vanish in virtue of the boundary conditions. Now each of the corpuscular world lines $L_{A}, L_{B}$ is, by assumption, isolated in the neighbourhoods of the boundaries $t=t_{1}$, $t=t_{2}$, of $\mathrm{R}$, and hence the considerations of $\$ 4$ apply to the field distributions about $\mathrm{L}_{\mathrm{A}}, \mathrm{L}_{\mathrm{B}}$ in these neighbourhoods. On making the transition $\kappa \rightarrow \infty$ to the corpuscular idealisation we may therefore express the left-hand side of (7.2) in terms of the energy-momentum vectors $m_{\mathrm{A}}^{i}, m_{\mathrm{B}}^{i}$ of the two corpuscles $\mathrm{A}, \mathrm{B}$ at the events in which their world lines $L_{A}, L_{B}$ intersect the temporal boundaries of $R_{4}$; equation (7.2) then yields

where

$$
\left[m_{\mathrm{A}}^{i}+m_{\mathrm{B}}^{i}\right]_{1}^{2}=\int_{t_{\mathrm{L}}}^{t_{2}} f^{i}(t) d t
$$

$$
f^{i}(t) \equiv-\lim _{\kappa \rightarrow \infty} \int\left\{\begin{array}{c}
i \\
j k
\end{array}\right\} T_{\kappa}^{j k} d V .
$$

It is to be noted that these equations, relating the total energymomentum before the impact to that after the impact, are not vectorial equations in the true sense, for they involve quantities computed at different events of $R$, and the manner in which these events are chosen is dependent on the coordinate system employed. Indeed, we can refer to them as dealing with the total energy and momentum only with reference to that privileged set of virtual observers $\mathrm{O}, x^{a}=$ const., signalised by that particular Gaussian coordinate system $x^{i}$ employed in setting up the metric (2.1). However, this situation is no cause for concern, for we are actually here only interested in what happens in the immediate vicinity of the event $\mathrm{E}\left(\xi^{i}\right)$ at which the collision takes place; the behaviour of each vector $m^{i}$ along the remainder of the paths $L$ within $R$, on which each of the particles may be regarded as isolated, is already determined by the considerations of the preceding $\$ \S 5,6$. Our investigation will thus be complete if we confine ourselves here to the situation in the neighbourhood of $\mathrm{E}$. 
In order to obtain a precise formulation, sufficient for the physical situations in mind, we assume that each energy-momentum vector $m^{i}$ possesses a limit $\left[m^{i}\right]_{+},\left[m^{i}\right]_{-}$as we approach the singular event $\mathbf{E}$ from the positive, and from the negative, $t$-directions, respectively, and that $f^{i}(t)$ is a bounded function of $t$ in a region containing $\mathbf{E}$. As we then allow $t_{1}, t_{2} \rightarrow \xi^{0}$ the right-hand side of (7.3) approaches the limit 0 , and we are left with the condition

$$
\left[m_{\mathrm{A}}^{i}+m_{\mathrm{B}}^{i}\right]_{+}=\left[m_{\mathrm{A}}^{i}+m_{\mathrm{B}}^{i}\right]_{-} ;
$$

i.e. the total energy and total momentum of the system are unchanged by the collision. This vectorial result, which refers only to the situation at a single event $\mathrm{E}$, is clearly applicable to the case in which any number of corpuscles collide at a single event.

It has not been found necessary to specify more precisely the nature of the corpuscles suffering the collision; they may be either material particles or photons, indescriminately. Thus if one, say A, is a photon which interacts with a particle $B$, we have the situation contemplated in the Compton effect, and the result (7.5), together with Planck's relation (6.10), leads to the more detailed theory of this effect. We remark, in closing, that the acceptance of the results of R. S. Shankland ${ }^{1}$ on the failure of this theory, would seem to entail some limitation on the possibility of a corpuscular description of the field which is more fundamental than those resulting from the usually accepted undulatory theory touched upon in the Introduction-or perhaps the eventual surrender of the field laws (1.3) themselves.

1 Phys. Rev. 49, p. 8 (1936). Note added in proof: This eventuality seems now to be removed, by the later work of Shankland, Phys. Rem. 50, p. 571 (1936), and of others. 